\title{
Erratum
}

\section{Erratum: Kota et al., "Hippocampal Theta Oscillations Support Successful Associative Memory Formation”}

In the article, "Hippocampal Theta Oscillations Support Successful Associative Memory Formation," by Srinivas Kota, Michael D. Rugg, and Bradley C. Lega, which appeared on pages 9507-9518 of the December 2, 2020 issue, a typographical error was made in the abstract. The online version has been updated to remove the extraneous word "fails" from the second sentence: "For this reason, the contrast between study items that are later recollected with their associative pair versus those for which no association is made should reveal electrophysiological patterns in the hippocampus selectively involved in associative memory encoding."

DOI: 10.1523/JNEUROSCI.3136-20.2020 